\title{
Optimal Path Planning for a Remote Sensing Unmanned Ground Vehicle in a Hazardous Indoor Environment
}

\author{
Mohammad R. Alenezi, Abdullah M. Almeshal \\ Electronics Engineering Technology Department, College of Technological Studies, PAAET, Shuwaikh, Kuwait \\ Email: am.almeshal@paaet.edu.kw
}

How to cite this paper: Alenezi, M.R. and Almeshal, A.M. (2018) Optimal Path Planning for a Remote Sensing Unmanned Ground Vehicle in a Hazardous Indoor Environment. Intelligent Control and $\mathrm{Au}$ tomation, 9, 147-157.

https://doi.org/10.4236/ica.2018.94011

Received: October 19, 2018

Accepted: November 12, 2018

Published: November 15, 2018

Copyright (c) 2018 by authors and Scientific Research Publishing Inc. This work is licensed under the Creative Commons Attribution International License (CC BY 4.0).

http://creativecommons.org/licenses/by/4.0/

\begin{abstract}
Urban search and rescue robots are playing an increasingly important role during disasters and with their ability to search within hazardous and dangerous environments to assist the first respond teams. Surveying and remote sensing the hazardous areas are two of the urgent needs of the rescue team to identify the risks before the intervention of the emergency teams. With such time-critical missions, the path planning and autonomous navigation of the robot is one of the primary concerns due to the need of fast and feasible path that is comprehensive enough to assess the associated risks. This paper presents a path planning method for navigating an unmanned ground vehicle within in an indoor hazardous area with minimum priori information. The algorithm can be generalized to any given map and is based on probabilistic roadmap path planning method with spiral dynamics optimization algorithm to obtain the optimal navigating path. Simulations of the algorithm are presented in this paper, and the results promising results are illustrated using Matlab and Simulink simulation environments.
\end{abstract}

\section{Keywords}

Remote Sensing, Path Planning, UGV, Autonomous Mobile Robot

\section{Introduction}

Urban search and rescue (USR) are time-critical missions, failing to find and rescue the victims in time within the hazardous areas may lead to a tragedy. Search and rescue robots have been proven to be useful in disasters such as hurricanes, volcanos, collapses and earth quakes [1] [2] [3]. Due to the need of a low-risk solution to detect the victims and assess the hazards, robots were 
equipped with sensing elements and have been utilized to navigate within the disastrous areas for searching and surveying [1] [2]. Whether manually tele operated or autonomously driven, various types of robots such as unmanned ground vehicles (UGV), unmanned aerial vehicles (UAV) and unmanned surface vehicles (USV), have been used in urban search and rescue missions [4] [5] [6]. Furthermore, first responders' teams have used robots to drop emergency kits at victims who are drowning in sea or trapped within hazardous areas such as mine collapses or under the rubbles after earth quakes [7] [8].

Surveying and remote sensing in an urban hazardous indoor area is a challenging problem due to the restricted accessibility for personnel and robots. Indoor navigation is difficult for robots due to the loss of GPS signals and would require a localization system to position the robot within the indoor environment [9] [10]. Synchronous Localization and Mapping (SLAM) methods would require more processing time and would be beneficial in less critical applications than search and rescue missions where time factor is of utmost importance to facilitate the aid and save victims lives [8] [10] [11]. This paper presents a novel work on combining path planning using SDA algorithm in an unknown indoor environment with minimum priori information.

This paper presents an approach for optimal path planning for a remote sensing autonomous robot in a cluttered and hazardous indoor environment. The operating scenario of this robot is applicable during the search and rescue missions, where unmanned ground vehicles (UGVs) are favored, to survey and sense the environment for various detectable phenomena such as gases, fire or smoke detection and etcetera. The proposed algorithm can be generalized to any given map and as an example simulation scenario; we present an application of path planning of a mobile robot in an urban search and rescue mission to navigate through an indoor hazardous building for remote-sensing and assessment of the hazardous situation. The sensing of the environment would enable the first responders' team to determine the severity of the emergency and would help them to decide on rescuing the victims with the least risk towards the team. With the proposed system, the robot will navigate autonomously by utilizing probabilistic roadmaps (PRM) to find out all the possible navigation paths for autonomous navigation of the robot, given the building map. With the various solutions of the probabilistic roadmaps, an optimal path that would be selected, based on particle swarm optimization algorithm, that covers most of the indoor area to provide the best possible assessment of the hazard situation.

\section{Probabilistic Roadmaps Path Planner}

Probabilistic roadmap (PRM) methods have been known for their efficient approach in path planning complex motions for a wide discipline of applications including various types of robots such as manipulators, unmanned robotic vehicles as well as predicting the motion and transitions of biological systems such as proteins and molecules [12] [13]. In robotics, PRM solves complex motion 
planning problems for a single or multiple robots with multiple degrees of freedom in free space.

PRM is based on representing an approximation of the free space (F) in a sample-based approach that is referred to as a configuration space and is composed of nodes and local paths or segments. The approximation of the free space is based on a probability measure to avoid computing an exact shape of the free space, thus the "probabilistic" term of the method. PRM algorithm is based on two steps; roadmap construction and the roadmap query. The algorithm requires start and goal points to calculate collision-free paths from the constructed representation of the free space. The basic PRM pseudocode used in this research is presented in Table 1.

With its simple pseudo code, the PRM algorithm could calculate many feasible paths in any given map. However, with the limited and constrained time of the rescue missions, an optimal path among all the calculated paths would be needed within a fast and reliable time to facilitate the given tasks of surveying or remote-sensing of the environment. Thus, a fast optimization algorithm would be needed to obtain an optimal comprehensive path with the given constraints.

\section{Spiral Dynamic Optimization Algorithm}

Spiral dynamics optimization algorithm (SDA) has been inspired by the common feature of the logarithmic spirals found in nature such as whirling currents and was introduced by Tamura and Yasuda [14] who believed that it could be of a beneficial search strategy. SDA is a relatively new metaheuristic optimization algorithm that was tested and compared against other common optimization methods such as particle swarm optimization (PSO) and bacterial foraging algorithm (BFA) and has shown an equal or better performance in terms of the speed of convergence and evolution of cost accuracy [15] [16].

The strength of the algorithm relies in the diversification and intensification of the search stages that mimics the whirling current spiral where the diversification covers a wider area of search and the intensification improves the cost accuracy around good solutions. With its fast convergence towards optimal cost

Table 1. Basic PRM pseudocode [13].

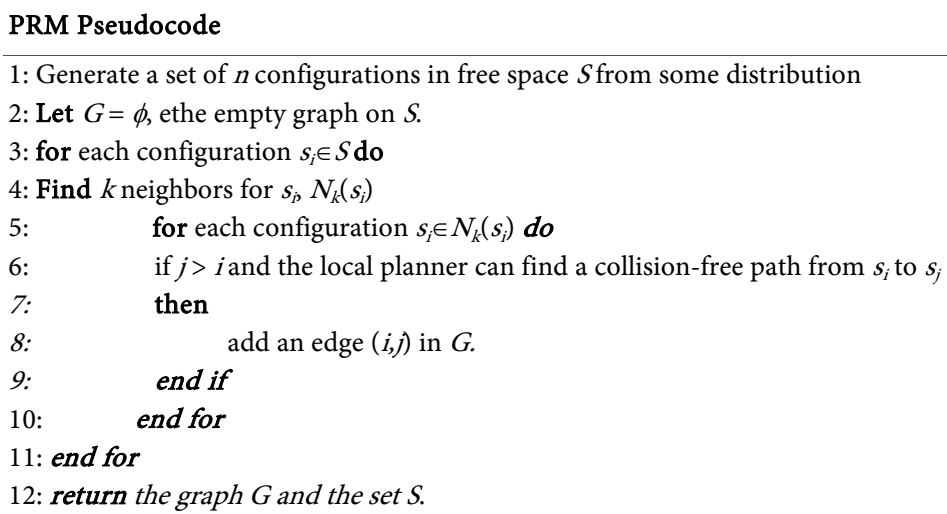


functions, SDA would enhance the search for optimal path among all the calculated PRM paths and would result in the optimal navigation path. The nomenclature of the SDA optimization is presented in Table 2 followed by the pseudocode of the algorithm in Table 3 as reported by Tamura and Yasuda in [14].

The rotation matrix for the $\mathrm{n}$-dimension SDA algorithm is defined as

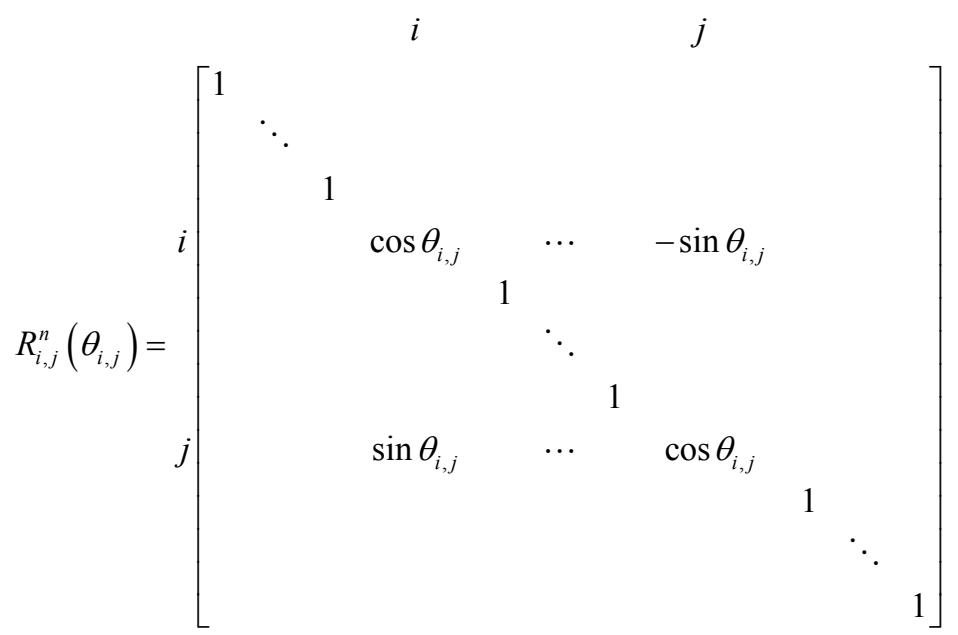

The $\mathrm{n}$-dimension spiral dynamic model is expressed using the rotational matrix as:

$$
x_{i}(k+1)=S_{n}(r, \theta) x_{i}(k)-\left(S_{n}(r, \theta)-I_{n}\right) x^{*}
$$

where

$$
S_{n}(r, \theta) x(k)=\prod_{i=1}^{n-1}\left(\prod_{j=1}^{i} R_{n-i, n+1-j}^{n}\left(\theta_{n-i, n+1-j}\right)\right)
$$

The simple structure both the PRM and SDA algorithms and would enable having an onboard processing unit to calculate the optimal path online. In the upcoming section, we demonstrate the feasibility of the proposed algorithm over a given operating scenario that involves an indoor cluttered map with obstacles.

\section{Simulations and Results}

The simulation scenario assumed in this paper is to have the robot navigate through some hazardous chemical laboratories where a gas leak has been detected. Figure 1 illustrates the building map with a total area of $(410 \times 280)$ square meters. The robot will be simulated to have a starting point at the reception

Table 2. SDA optimization nomenclature.

\begin{tabular}{cl}
\hline Parameter & \\
\hline$\theta$ & Rotation angle, $0 \leq \theta \leq 2 \pi$ \\
$k_{\max }$ & Maximum iteration number. \\
$\mathrm{r}$ & Convergence rate of distance between a point and the origin, $0 \leq r \leq 1$ \\
$R_{i, j}$ & Rotation matrix between $x i-x j$ planes \\
$\mathrm{m}$ & Dimension of the search space \\
\hline
\end{tabular}


Table 3. SDA optimization pseudocode [14].
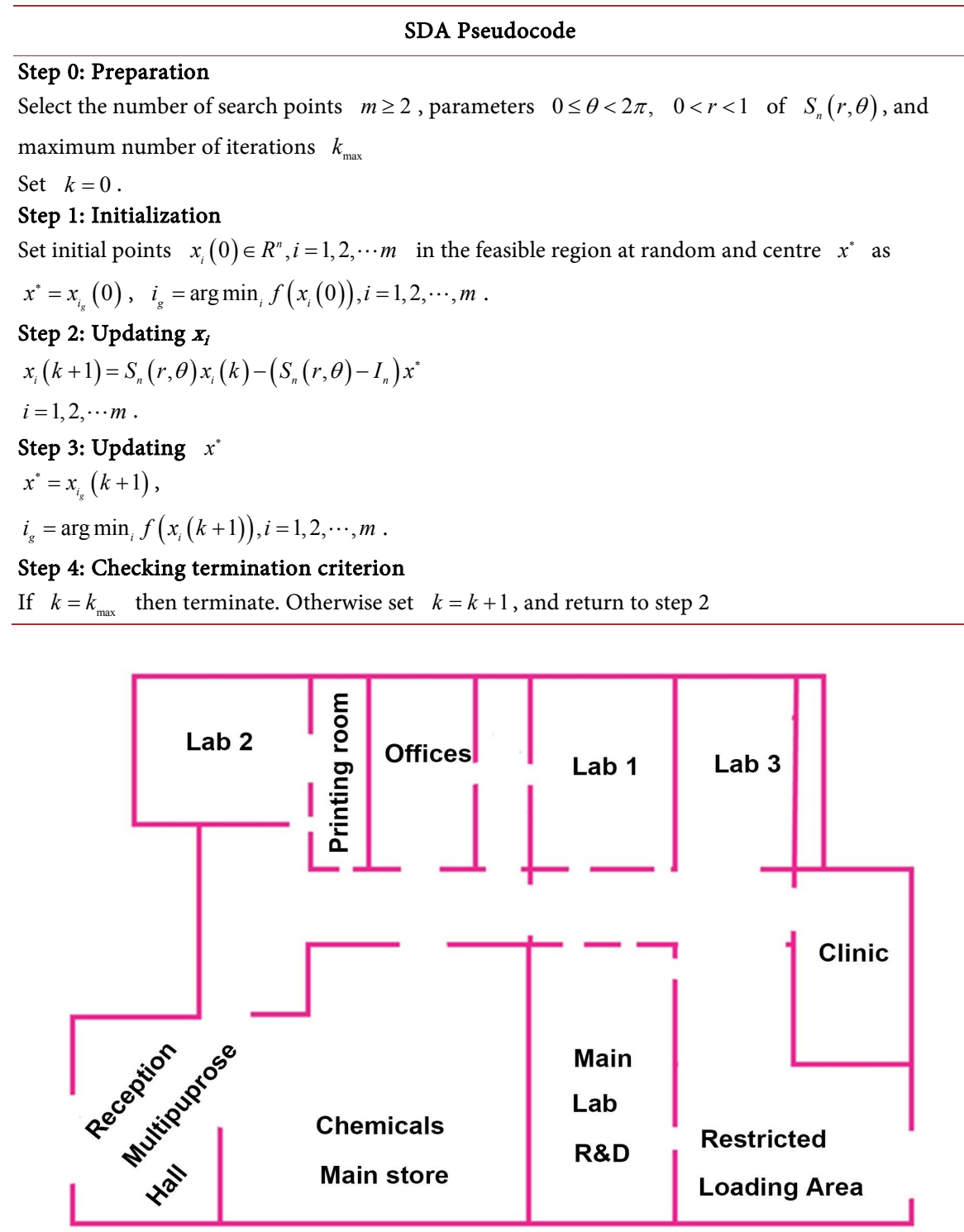

Figure 1. Chemical Laboratories building map.

and autonomously navigate throughout the building corridors to reach the exit at the restricted loading area as in Figure 1. The building is assumed to have a cluttered environment with many obstacles and barriers within the navigation path such as furnishing, equipment and building infrastructures as illustrated in Figure 2. These obstacles would need to be defined for the path planning algorithm to be avoided and an optimal path is calculated.

To start simulating the PRM algorithm, the building map need to be converted into binary occupancy grid matrices in order to define the area constraints for the algorithm. Figure 3 presents the converted map that is drawn using a binary occupancy grid matrix.

Simulations start with the SDA optimization algorithm to find out the best navigating path where the robot can record most of the sensory data throughout 


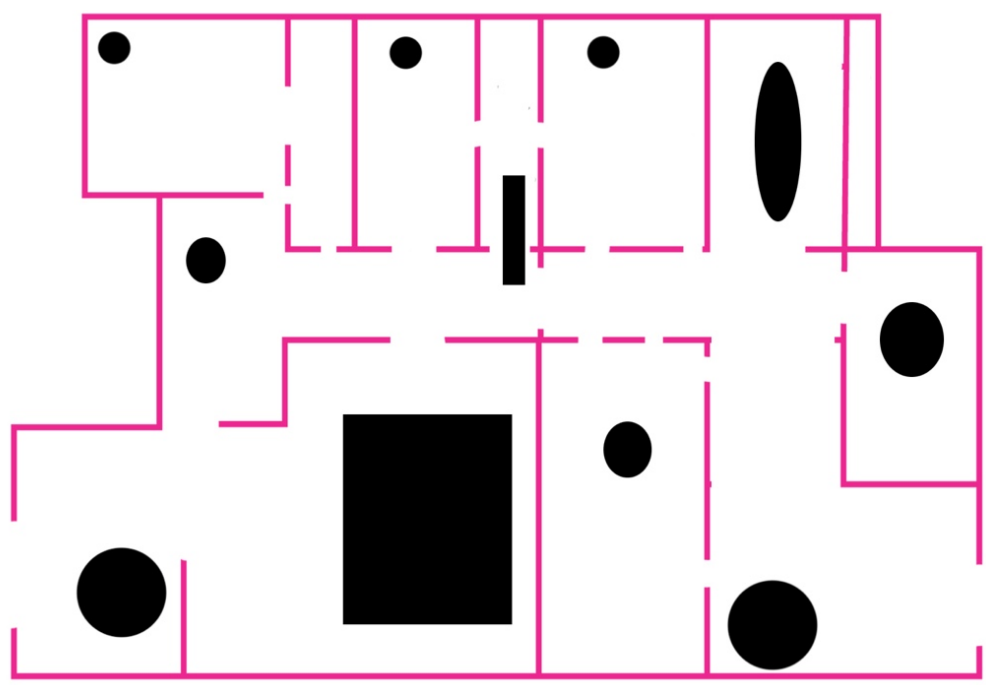

Figure 2. The obstacles and barriers of the building.

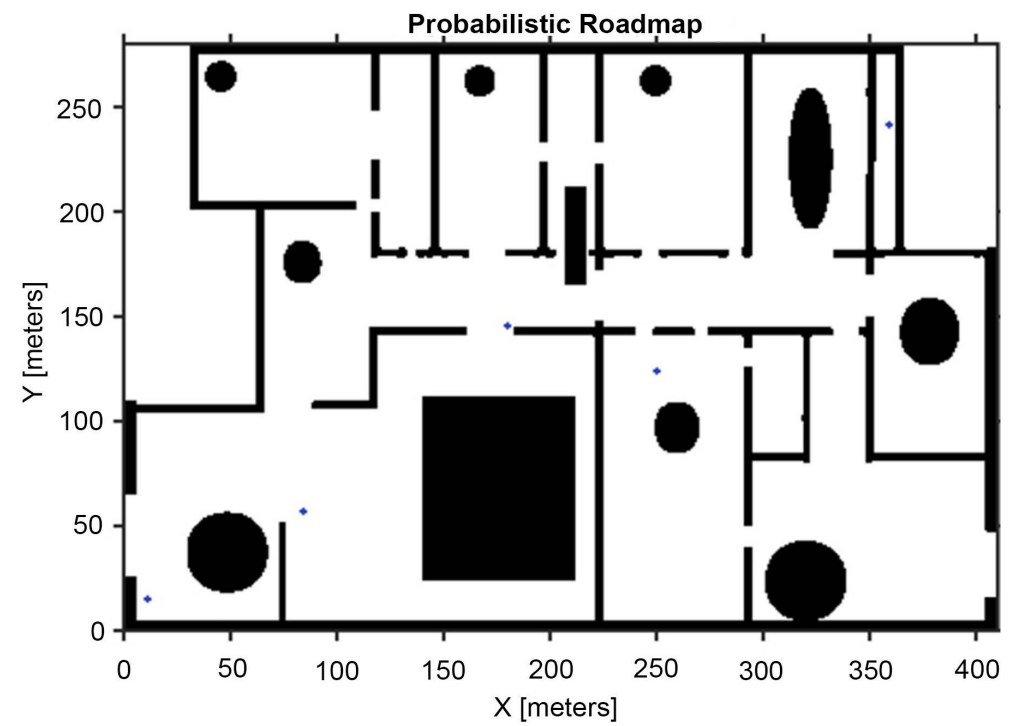

Figure 3. A grayscale map constructed from the binary occupancy grid.

the corridors until reaching the end of the path. The objective function is defined to have the best connection distance between the PRM nodes. Table 4 presents the simulation constraints in terms of the number of nodes and connection distances.

Figure 4 and Figure 5 show the lower and upper limits of the PRM results respectively with various numbers of nodes and connection distances. With the lower limits of the PRM parameters, as shown in Figure 4(a), the PRM has failed to plan a path due to the low number of connecting nodes. On the other hand, the PRM has found a possible path from the proposed starting point till the end point but without passing through all the possible corridors of the building as shown in Figure 4(b). Thus, failing to record the sensory readings and may lead to a poor decision of the responding team. The SDA algorithm is simulated with the following parameters: (Table 5) 
Table 4. Simulation constraints of PRM algorithm parameters.

\begin{tabular}{ccc}
\hline Parameter & Lower limit & Upper limit \\
\hline Number of nodes & 80 & 700 \\
Connection distance & 50 & 200 \\
\hline
\end{tabular}

Table 5. SDA Simulation parameters.

\begin{tabular}{cc}
\hline Parameter & Value \\
\hline $\mathrm{P}$ & 15 \\
$\mathrm{R}$ & 0.95 \\
$\theta$ & $\frac{\pi}{4}$ \\
Initial points & 10 \\
Iterations & 250 \\
\hline
\end{tabular}

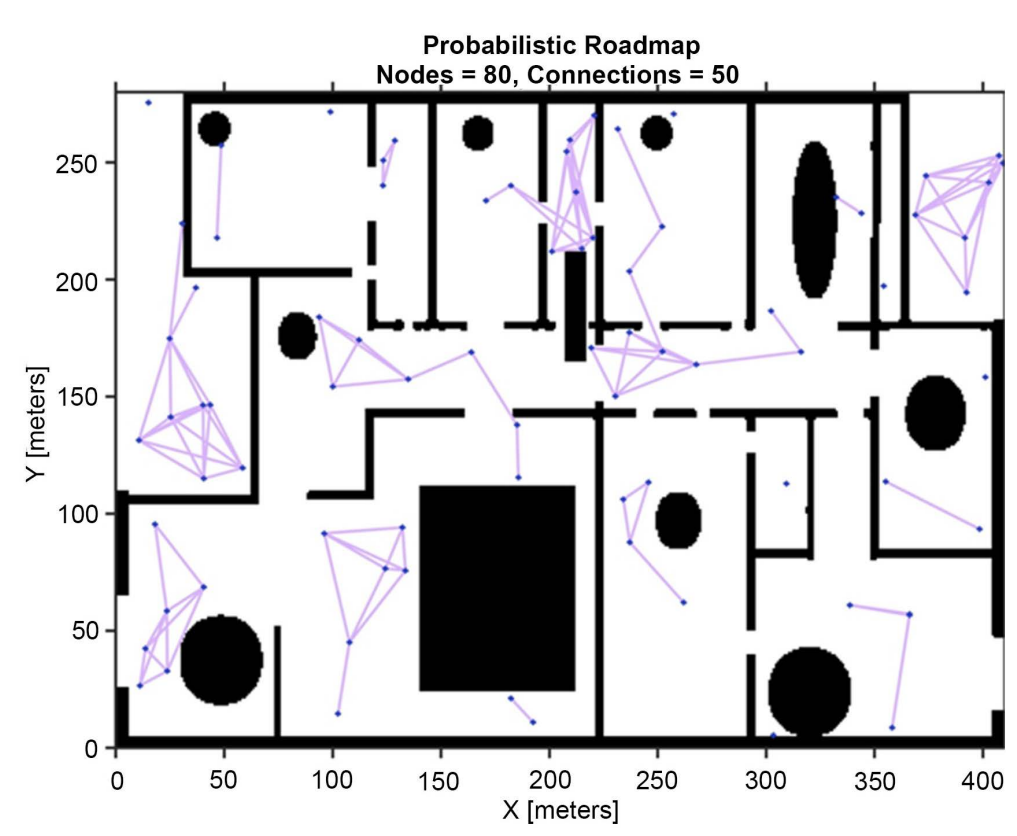

Figure 4. Path planner simulation with the lower limits of the PRM parameters.

The SDA has converged to the optimum number of nodes of 419 within 250 iterations as shown in Figure 6. Numerous simulations were carried out to observe the convergence of the SDA solution towards the optimal path. Figure 7 illustrate simulation trials before the convergence to the optimal solution of the path planning.

The optimal path is therefore presented in Figure 8 with the optimized number of nodes of the PRM. As it can be clearly observed from Figure 6 that the optimized number of nodes by the SDA algorithm has resulted in a better navigating path that covers most of the corridors when compared to the previous trials of Figure 7. In addition, the PRM has successfully calculated a navigation path of the robot and avoided the obstacles and barriers within the environment. 


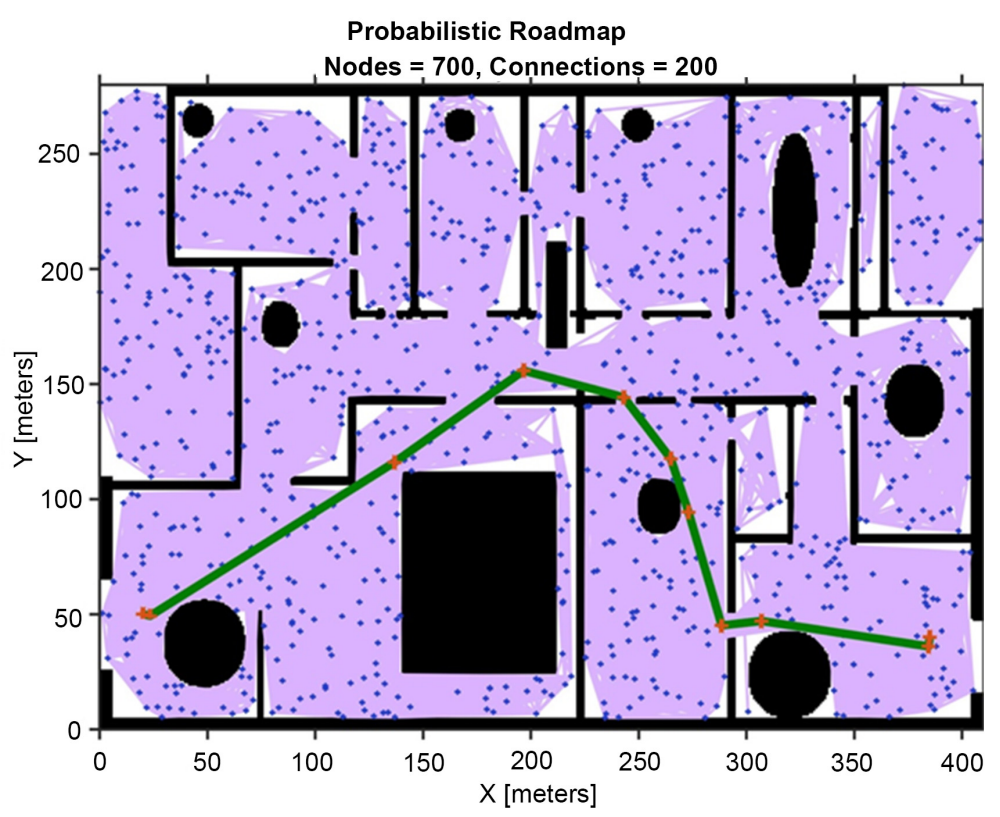

Figure 5. Path planner simulation with the upper limits of the PRM parameters.

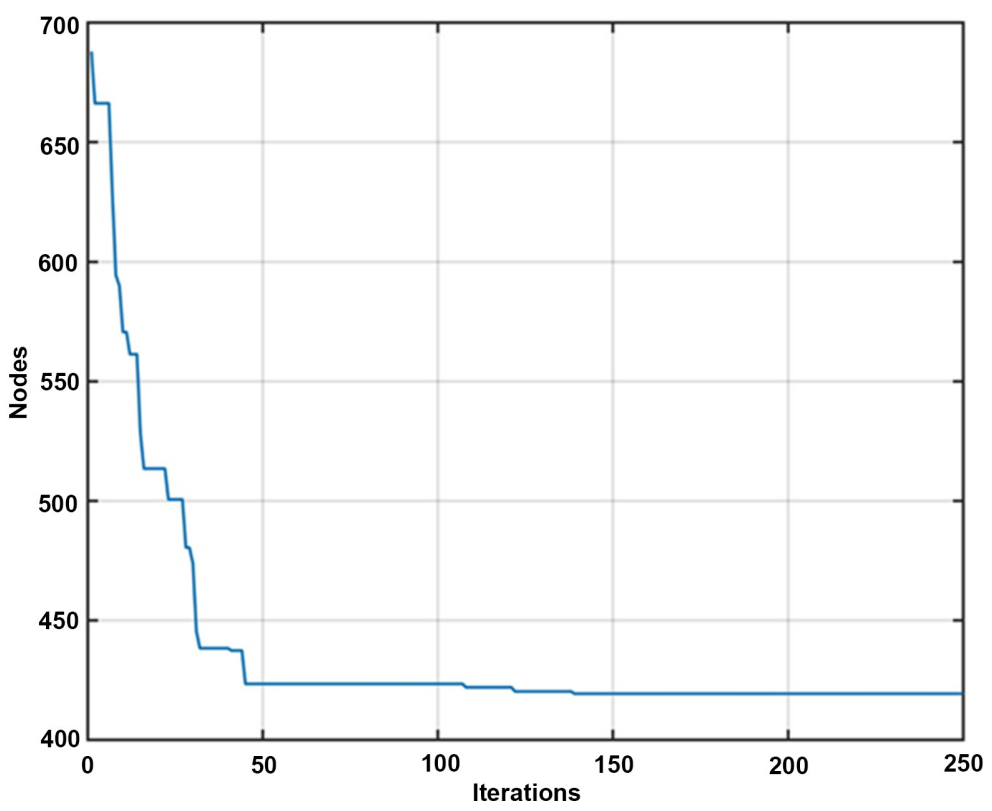

Figure 6. Path planner simulation with the upper limits of the PRM parameters.

Therefore, leading to more precise sensory measurements for the responders' team.

\section{Conclusions}

This paper set out with the aim of providing a fast and reliable optimal path planning algorithm for a remote-sensing unmanned ground vehicle in an indoor hazardous environment. A proposed path planning algorithm consisting of PRM and SDA optimization algorithm has been presented. The previous section has 


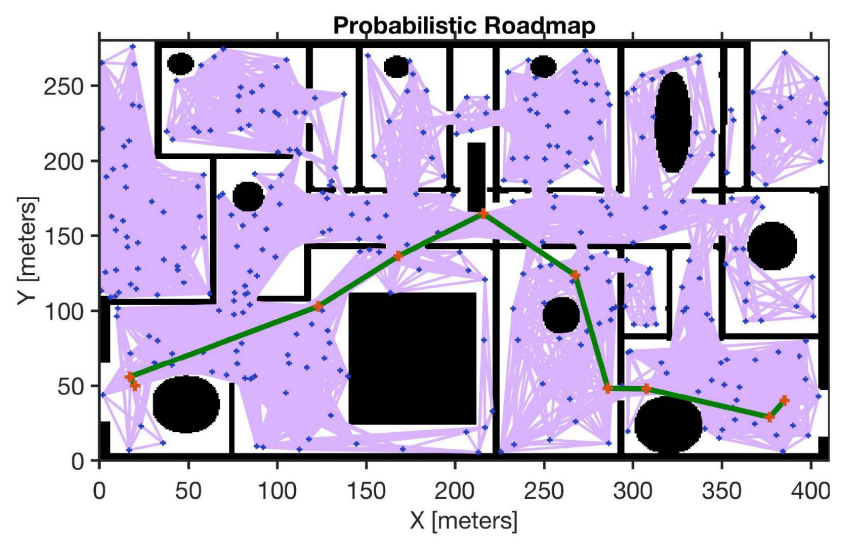

(a)

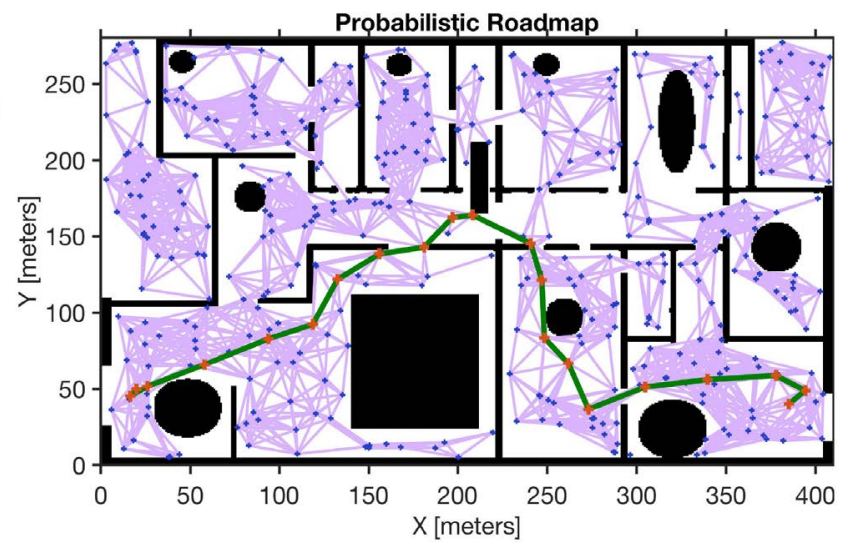

(b)

Figure 7. An example of two non-optimal solution illustrated by the SDA algorithm trial whilst searching for the best path within the given constraints.

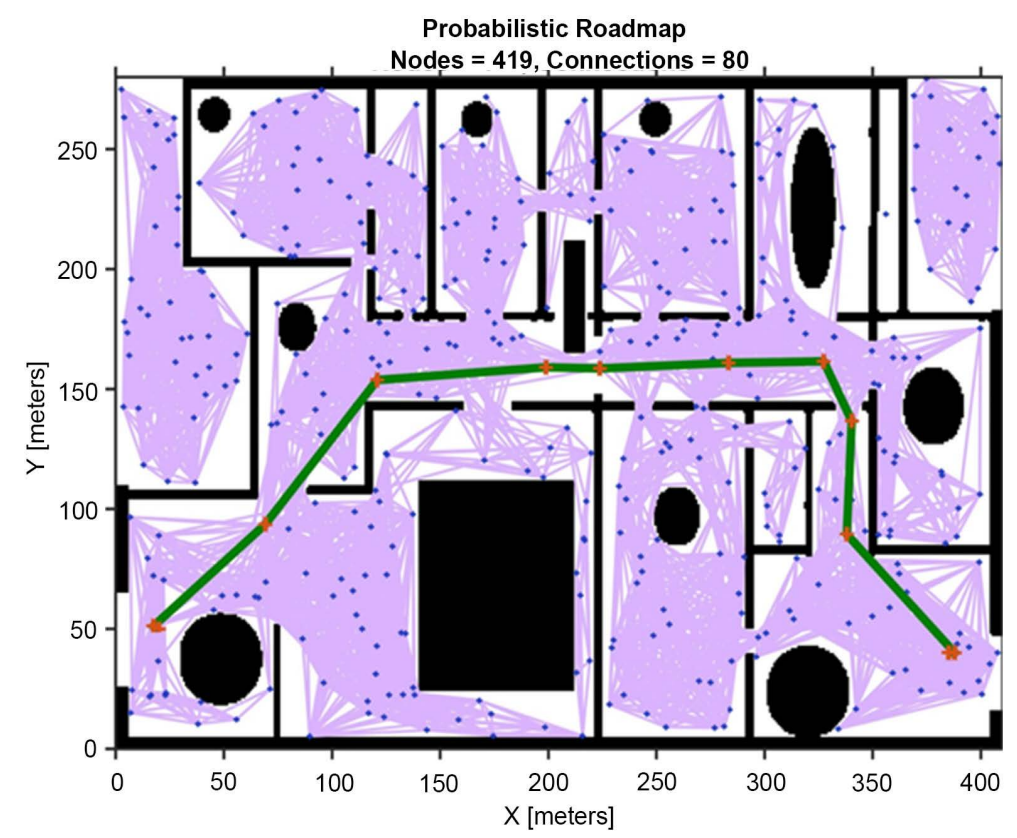

Figure 8. Optimal navigation path obtained by the PRM and SDA algorithms.

illustrated the feasibility of the proposed algorithm and has demonstrated promising results in a simulation scenario of a hazardous indoor environment.

These simulation results provide further support to be tested experimentally on an unmanned ground vehicle with an onboard processing unit and remote sensing equipment to get an insight how the complexity of a given map would affect the online processing time as well as for further verification of the algorithm. Further experimental studies of the algorithm are therefore recommended.

\section{Conflicts of Interest}

The authors declare no conflicts of interest regarding the publication of this paper. 


\section{References}

[1] Lermen, Z., Bettinger, E., Drinks, D., Carter, C., Culp, T., Martinez, D. and Stafford, M. (2016) Search and Rescue Robot. Florida institute of Technology, Melbourne.

[2] Horsch, C., Cuijpers, I.R. and Ham, J. (2012) Urban Search and Rescue Robots. Eindhoven University of Technology, Eindhoven.

[3] Davids, A. (2002) Urban Search and Rescue Robots: From Tragedy to Technology. IEEE Intelligent Systems, 17, 81-83. https://doi.org/10.1109/MIS.2002.999224

[4] Saddington, P. and Ramchurn, S.D. (2018) The Multimodal Speech and Visual Gesture (mSVG) Control Model for a Practical Patrol, Search, and Rescue Aerobot. Towards Autonomous Robotic Systems: 19th Annual Conference, TAROS 2018, Bristol, 25-27 July 2018, 423.

[5] Sampedro, C., Rodriguez-Ramos, A., Bavle, H., Carrio, A., de la Puente, P. and Campoy, P. (2018) A Fully-Autonomous Aerial Robot for Search and Rescue Applications in Indoor Environments using Learning-Based Techniques. Journal of Intelligent \& Robotic Systems, 91, 1-27.

[6] Goldhoorn, A., Garrell, A., Alquézar, R. and Sanfeliu, A. (2018) Searching and Tracking People with Cooperative Mobile Robots. Autonomous Robots, 42, 739-759. https://doi.org/10.1007/s10514-017-9681-6

[7] Wong, C., Yang, E., Yan, X.T. and Gu, D. (2018) Autonomous Robots for harsh Environments: A Holistic Overview of Current Solutions and Ongoing Challenges. Systems Science \& Control Engineering, 6, 213-219. https://doi.org/10.1080/21642583.2018.1477634

[8] Roy, N., Abedin, M.I., Sanila, S.K., Ahmed, S., Saumik, S.S. and Rahman, M.M. (2018) Robotic Unit Model Proposal for Rescue \& Aiding: For Disasters in Bangladesh. 2018 International Conference on Computer, Communication, and Signal Processing (ICCCSP), Chennai, 22-23 February 2018, 1-5.

[9] Colomina, I. and Molina, P. (2014) Unmanned Aerial Systems for Photogrammetry and Remote Sensing: A Review. ISPRS Journal of Photogrammetry and Remote Sensing, 92, 79-97. https://doi.org/10.1016/j.isprsjprs.2014.02.013

[10] Rossi, M., Brunelli, D., Adami, A., Lorenzelli, L., Menna, F. and Remondino, F. (2014) Gas-Drone: Portable Gas Sensing System on UAVs for Gas Leakage Localization. SENSORS, 2014 IEEE, Valencia, 2-5 November 2014, 1431-1434. https://doi.org/10.1109/ICSENS.2014.6985282

[11] Cook, G. (2011) Mobile Robots: Navigation, Control and Remote Sensing. John Wiley \& Sons, New York City. https://doi.org/10.1002/9781118026403

[12] Saha, M. (2006) Motion Planning with Probabilistic Roadmaps. Doctoral Dissertation, Stanford University, Stanford.

[13] Bekris, K.E., Chen, B.Y., Ladd, A.M., Plaku, E. and Kavraki, L.E. (2003) Multiple Query Probabilistic Roadmap Planners using Single Query Primitives. IEEE, Piscataway.

[14] Tamura, K. and Yasuda, K. (2011) Primary Study of Spiral Dynamics Inspired Optimization. IEEJ Transactions on Electrical and Electronic Engineering, 6, S98-S100. https://doi.org/10.1002/tee.20628

[15] Almeshal, A.M., Goher, K.M., Nasir, A.N.K., Tokhi, M.O. and Agouri, S.A. (2013) Fuzzy Logic Optimized Control of a Novel Structure Two-Wheeled Robotic Vehicle using HSDBC, SDA and BFA: A Comparative Study. 2013 18th International Conference on Methods \& Models in Automation \& Robotics (MMAR), Miedzyzdroje, 26-29 August 2013, 656-661. https://doi.org/10.1109/MMAR.2013.6669988 
[16] Goher, K.M., Almeshal, A.M., Agouri, S.A., Nasir, A.N.K., Tokhi, M.O., Alenezi, M. R., Fadlallah, S.O., et al. (2017) Hybrid Spiral-Dynamic Bacteria-Chemotaxis Algorithm with Application to Control Two-Wheeled Machines. Robotics and Biomimetics, 4, 3. https://doi.org/10.1186/s40638-017-0059-1 